\title{
Treatment effect of cyclosporine $A$ in patients with painful bladder syndrome/interstitial cystitis: A systematic review
}

\author{
ZHIKUI WANG and LEI ZHANG \\ Department of Nephrology, Linyi People's Hospital, Linyi, Shandong 276003, P.R. China
}

Received January 25, 2015; Accepted February 4, 2016

DOI: 10.3892/etm.2016.3301

\begin{abstract}
Cyclosporine A (CyA) is emerging as a potential therapeutic strategy for painful bladder syndrome/interstitial cystitis (PBS/IC), which is currently an incurable disease. The purpose of this systematic review was to evaluate the treatment effects of CyA in PBS/IC. Electronic and manual retrieval procedures were carried out to identify eligible references for the systematic review. The entire contents of the included articles were assessed, from study design to reported results. Eight studies, comprising three randomized controlled trials (RCTs), four prospective studies and one retrospective cohort study, were included, involving a total of 298 subjects. Meta-analysis was not implemented due to heterogeneity of the manner of reporting the outcome parameters. All studies reported an improvement in symptoms following treatment with CyA. The results of the three RCTs implied that the treatment effects of CyA were better than those of pentosan polysulfate sodium. Some adverse events, for example, elevation of serum creatinine levels and an increase in blood pressure, were noted in five studies. In conclusion, the evidence from the studies implied that treatment of CyA can result in a long-term benefit in patients of $\mathrm{PBS} / \mathrm{IC}$; however, further evidence is required to verify this.
\end{abstract}

\section{Introduction}

Painful bladder syndrome/interstitial cystitis (PBS/IC) occurs far more frequently in women than in men. It has been reported that among 1.3 million Americans having the symptoms of PBS/IC, >1.2 million were women in the year 2007 (1). The typical age predilection is $\sim 40$ years (2). The common symptoms of PBS/IC include urinary urgency, frequency, nocturia and suprapubic or pelvic pain without any known etiological factor. Diagnostic criteria have been established by the National

Correspondence to: Professor Lei Zhang, Department of Nephrology, Linyi People's Hospital, 27 Jiefang Road, Linyi, Shandong 276003, P.R. China

E-mail: leizhang_phls@yeah.net

Key words: cyclosporine A, interstitial cystitis, painful bladder syndrome, systematic review, treatment effect
Institute of Diabetes and Digestive and Kidney Diseases (NIDDK) in order to standardize the diagnosis of PBS/IC; these include objective findings of glomerulations or Hunner's ulcer at cystoscopy and subjective symptoms of bladder pain or urinary urgency, in addition to multiple exclusion criteria such as age $<18$ years, duration of symptoms $<9$ months and absence of nocturia (3). Even though numerous treatments have been reported, including intravesical botulinum toxin $\mathrm{A}$ injections, pentosan polysulfate sodium (PPS), dietary modification, lifestyle interventions/behavioral therapies and bladder training, no specific effective therapy has yet been identified.

As an immunosuppressive agent, cyclosporine A (CyA) suppresses the activation of $\mathrm{T}$ cells by inhibiting the enzymatic activity of calcineurin (4). It has been successfully used in various conditions, such as in transplant recipients, hepatitis C (5), and autoimmune diseases such as psoriasis (6), Crohn's disease (7) and rheumatoid arthritis (8). Furthermore, CyA is emerging as a potential therapeutic strategy for PBS/IC, which is an intractable condition to treat. Several studies have reported promising results for the treatment effects of CyA in PBS/IC. However, the strength of the evidence of efficacy in each independent study is limited by various factors, such as limitation of included subjects, or absence of a control group. Therefore, the present systematic review was performed in order to assess the global evidence concerning the treatment effects of CyA in PBS/IC.

\section{Materials and methods}

Search strategy. The search strategy included the following key words variably combined: 'cyclosporine A,' 'bladder pain syndrome' and 'interstitial cystitis'. Databases including China National Knowledge Infrastructure (CNKI), PubMed, Highwire, EMBASE and Science Direct were searched for relevant references. In addition, other retrieval strategies were performed to screen for relevant articles, including manual retrieval and scanning of the reference sections of eligible articles.

Inclusion/exclusion criteria. Studies were regarded as qualified for inclusion if they met all the following inclusion criteria: i) the disease type was PBS/IC with or without meeting the NIDDK criteria; ii) the study was a clinical trial, including randomized controlled trials (RCTs), and prospective or retrospective cohort studies; iii) the treatment effects of CyA in 
PBS/IC were measured. Studies were excluded on the basis of any of the following criteria: i) the article was a review, letter or case report; ii) the article did not relate to the treatment effects of CyA in PBS/IC.

Data selection. Two researchers independently screened the initially included articles on the basis of the inclusion/exclusion criteria. Any disagreement was resolved by consensus. Baseline data and result information were extracted and assessed by the same researchers, including first author and publication year, country, inclusion/exclusion criteria of each study, subjects, gender (female/male), mean age, regimen protocol, outcome parameters, follow-up and complications. In addition, all included articles were assessed for methodological quality, according to the Newcastle-Ottawa scale (9) for nonrandomized studies and Jadad score for RCTs (10).

\section{Results}

Eligible articles. The various steps of the filtration procedure used in this review are presented in Fig. 1. Initially, 117 articles were included (26 from PubMed, 11 from CNKI, 36 from Highwire, 17 from EMBASE and 31 from ScienceDirect). No further relevant references were identified by manual search and filtration of the reference sections of included articles. Following careful screening of the titles and abstracts of each article, 95 articles were excluded, leaving 22 articles for further filtration. After screening the entire contents of these articles, 14 articles were further excluded. Eight articles (11-18) were finally included as eligible articles, which including a total of 298 subjects with a median number of 37.25 subjects per study. These 8 studies included 3 RCTs (16-18), 4 prospective cohort studies $(11-13,15)$ and 1 retrospective cohort study (14). In the 7 studies that reported the ages of the subjects, the age predilection was 40-70 years. In one of the articles (12), the patient age could not be identified because only the abstract was available. The included subjects were mostly females; the percentage of males was relatively low. The sample sizes of the included studies were uniformly small, ranging from 10 to 64. Other characteristics of the included articles are listed in Table I.

Six studies used the NIDDK criteria for diagnosing IC $(12,14,16-18)$; the other two studies used clinical symptoms to diagnose PBS/IC $(11,13)$. The subjects of two studies $(13,14)$ were patients in whom multiple first line therapies had failed. Regimen protocols were presented in all studies; the initial dosage of CyA was typically $2-3 \mathrm{mg} / \mathrm{kg}$ / day, sometimes divided into 2 daily dosages. If the symptoms were alleviated, the dosage of CyA was gradually decreased to a tolerance dose; if adverse events occurred the dose was reduced or the drug was stopped. In the three RCTs (16-18), the treatment therapy administered to the control group was $100 \mathrm{mg}$ PPS 4 times daily.

Methodological quality. All studies have detailed descriptions of the ascertainment of diagnosis, assessment of outcome and the instruments used to assess outcomes. All of the articles, with the exception of one (15), reported the follow-up time; the longest duration of follow-up was 5 years (12) and, the proportion of patients lost to follow-up

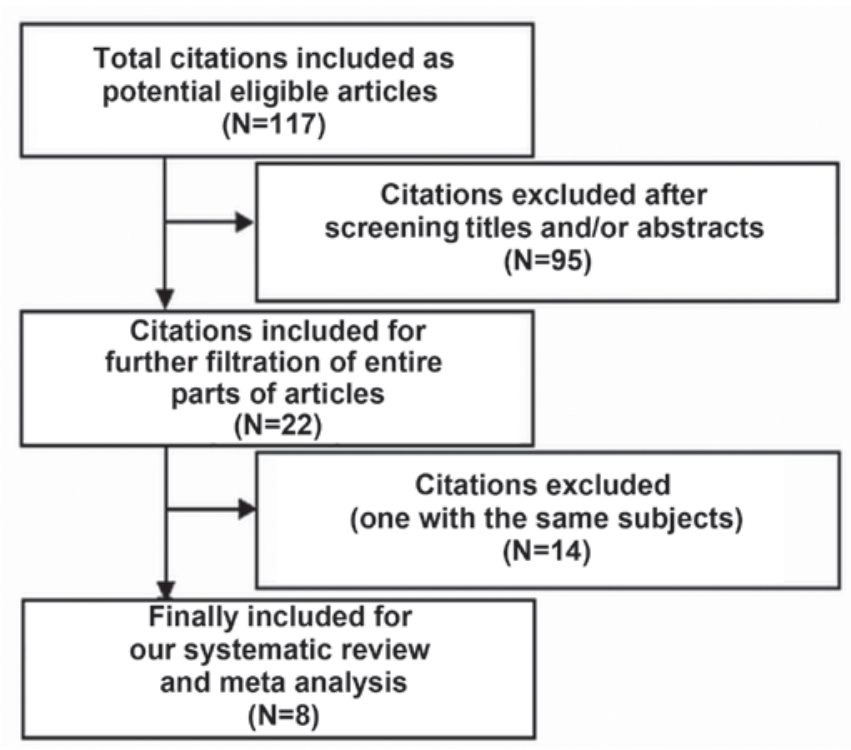

Figure 1. Reference retrieval procedure used in this systematic review of the treatment effect of cyclosporine A in patients with painful bladder syndrome/interstitial cystitis.

was $<20 \%$ in all studies. On the basis of the Jadad scale, the scores for the included RCTs ranged from 1 to 3 (regard as low methodological quality).

Outcome assessment. The outcome assessment of the included studies is presented in Table II. The O'Leary-Sant Symptom and Problem Indices of Interstitial Cystitis (ICSI and ICPI, respectively) were used as outcome measures in 5 studies $(11-13,16,17)$. Other outcome parameters were reported, including mean voided volume $(\mathrm{ml})(12,14-17)$, maximum awake bladder capacity (ml) (14-16), urinary frequency (24 h) (14-17), pain score (visual analog scale, VAS) $(16,17)$, cytokine levels $(11,17)$, daily urinary output (ml) (15), nocturia episodes (16) and improvement of quality of life (QoL) (18). All outcome parameters, with the exception of the biomarker IL-6, were reported to exhibit improvement following treatment of CyA, even though the degree of improvement varied. However, Sairanen et al (17) reported that IL-6 was significantly $(\mathrm{P}=0.04)$ decreased in patients $\geq 53$ years old following treatment with CyA, but not following treatment with PPS $(\mathrm{P}=0.73)$. All reported parameters in the three RCTs implied that the treatment effects of CyA were far superior to those of PPS treatment. In addition, long-term treatment with a low dosage of CyA could provide a persistent improvement of symptoms. The symptoms were recurrence if the treatment of CyA was discontinued.

Adverse effects. Five of the 8 included studies reported complications, which mainly comprised tiredness, abdominal pain, nausea, diarrhea and gingival hyperplasia (1 patient), induced hair growth (3 patients), serum creatinine elevation (12 patients) and a rise in blood pressure (8 patients). Following symptomatic treatment, the majority of patients were able to undergo sustained $\mathrm{CyA}$ treatment. However, 4 patients were required to stop treatment with CyA following increases in serum creatinine levels. 


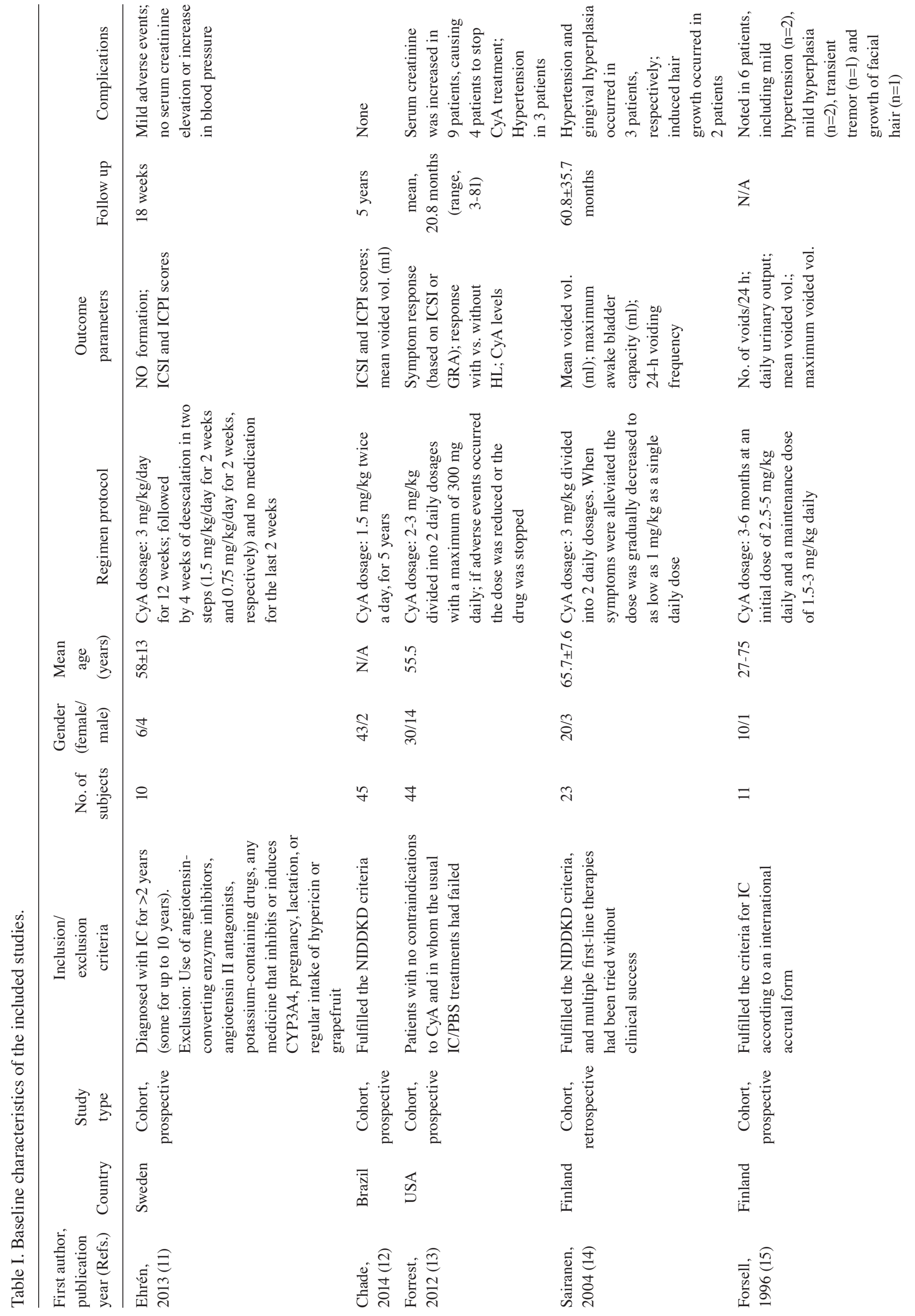




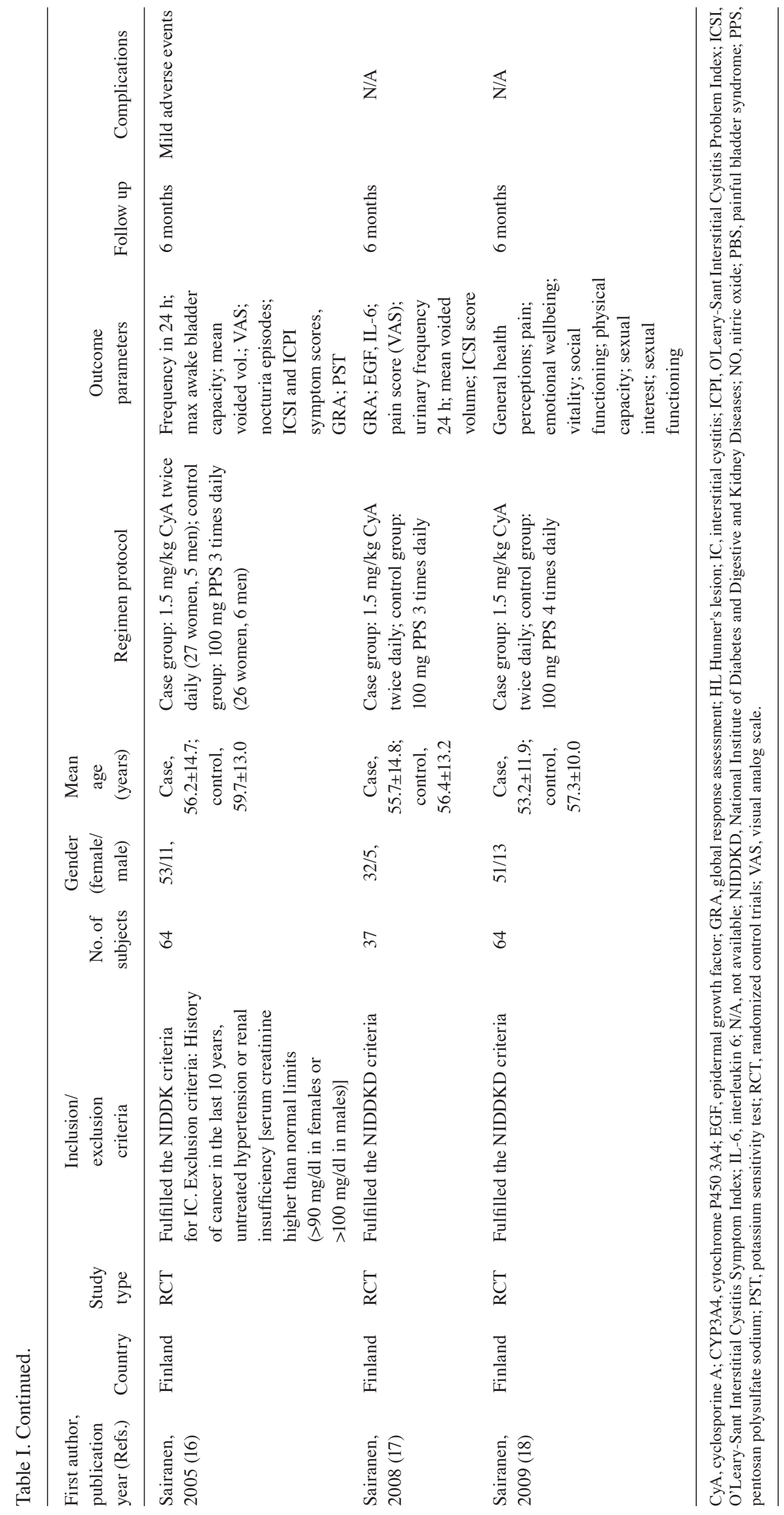




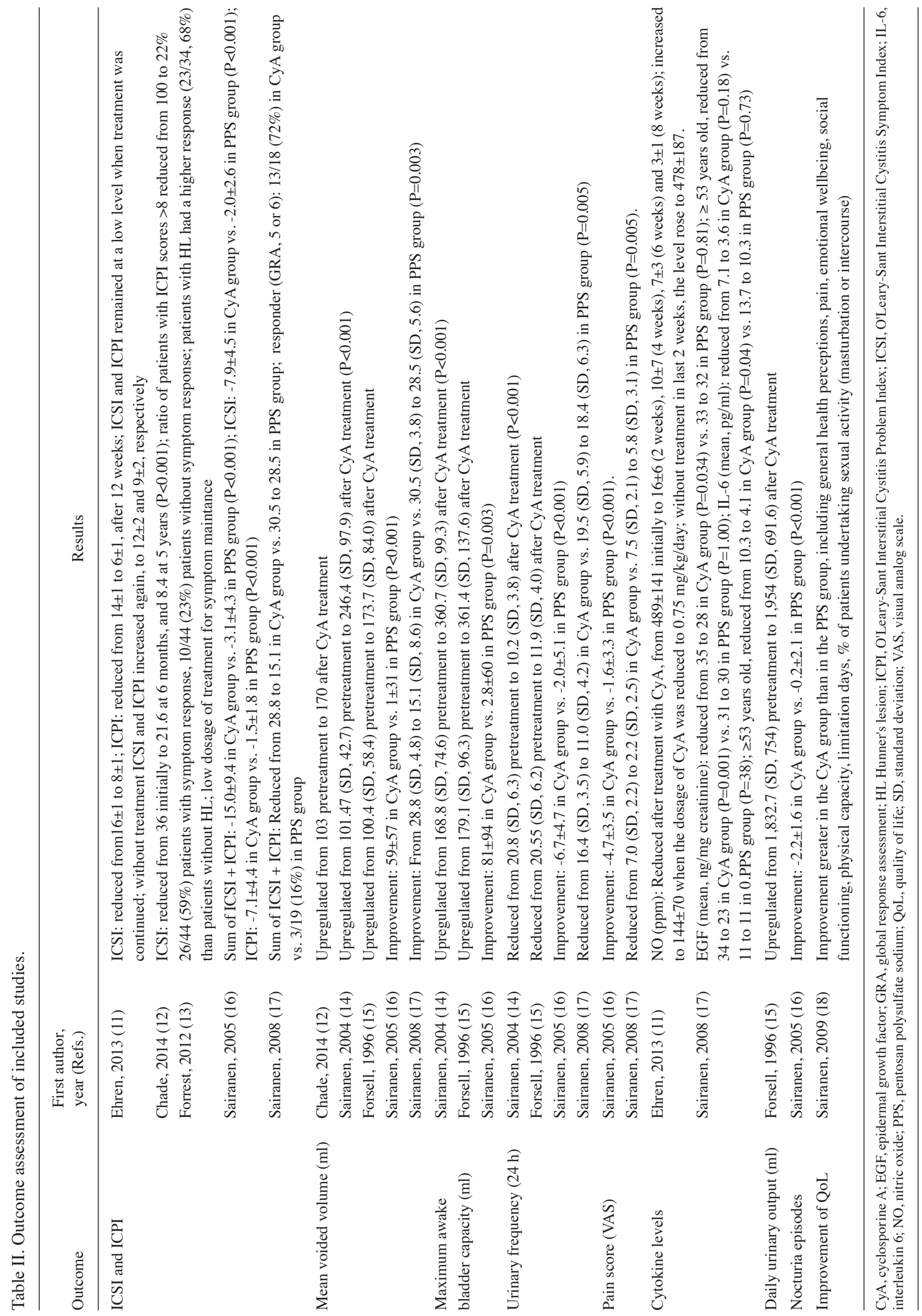




\section{Discussion}

The evidence from the included studies demonstrates that oral treatment with CyA may be an appropriate therapeutic strategy for patients with PBS/IC, and there is a trend towards benefit with a long-term low-dosage regimen. To the best of our knowledge, he present review summarizes all the prospective or retrospective non-randomized cohort trials and RCTs that exist on this topic.

CyA may be more advantageous in severe PBS/IC, because two studies $(11,13)$ included patients in whom the usual IC/BPS treatments had failed, and the symptoms and parameters in those patients improved following treatment with CyA (2-3 $\mathrm{mg} / \mathrm{kg}$ divided into 2 daily dosages with a maximum of $300 \mathrm{mg}$ daily). In addition CyA exhibited a higher curative effect than PPS. Long-term low-dosage CyA treatment can provide and maintain good symptom relief (19). However, symptoms may recur if the therapy with CyA is stopped. Hence, it is suggested that the use of CyA may be primarily as a drug for symptom improvement rather than a fundamental solution or cure. In addition, a series of adverse effects (in particular, upregulation of serum creatinine levels and hypertension) are of concern when oral CyA therapy is carried out.

There are several advantages of this systematic review. Firstly, a critical issue has been addressed. The search strategy was full-scale and without language restrictions. Secondly, two reviewers independently screened the eligible articles and extracted data with minimum errors. Thirdly, attempts were made to contact authors of published and unpublished studies to obtain relevant details for this systematic review.

However, the included studies contained small numbers of patients with short-term or long-term follow-up. For one included study (12), only the abstract was available for analysis in this systematic review, and it was not possible to obtain further details. The heterogeneity of the diagnosis criteria of PBS/IC, differences in methods, varied number of outcome measures employed and small number of participants, create challenges in the analysis of the treatment effect of CyA in PBS/IC. Therefore, further higher quality clinical trials are required to further explore the effectiveness of oral CyA in the treatment of PBS/IC.

\section{References}

1. Clemens JQ, Link CL, Eggers PW, Kusek JW, Nyberg LM and McKinlay JB; BACH Survey Investigators: Prevalence of painful bladder symptoms and effect on quality of life in black, Hispanic and white men and women. J Urol 177: 1390-1394, 2007.

2. Held PJ, Hanno PM, Wein AJ, Pauly MV and Cahn MA: Epidemiology of interstitial cystitis. In: Interstitial Cystitis. Hanno PM, Staskin DR, Krane RJ and Wein AJ (eds) Springer, London, pp29-48, 1990.
3. Gillenwater JY and Wein AJ: Summary of the National Institute of Arthritis, Diabetes, Digestive and Kidney Diseases workshop on interstitial cystitis, National Institutes of Health, Bethesda, Maryland, August 28-29, 1987. J Urol 140: 203-206, 1988.

4. Ogawa H, Kameda H, Amano K and Takeuchi T: Efficacy and safety of cyclosporine A in patients with refractory systemic lupus erythematosus in a daily clinical practice. Lupus 19: 162-169, 2010.

5. Forns $X$ and Navasa M: Cyclosporine A or tacrolimus for hepatitis C recurrence? An old debate. Am J Transplant 11: 1559-1560, 2011.

6. Maza A, Montaudiè H, Sbidian E, Gallini A, Aractingi S, Aubin F, Bachelez H, Cribier B, Joly P, Jullien D, et al: Oral cyclosporin in psoriasis: A systematic review on treatment modalities, risk of kidney toxicity and evidence for use in non-plaque psoriasis. J Eur Acad Dermatol Venereol 25 (Suppl 2): S19-S27, 2011.

7. Sternthal MB, Murphy SJ, George J, Kornbluth A, Lichtiger S and Present DH: Adverse events associated with the use of cyclosporine in patients with inflammatory bowel disease. Am J Gastroenterol 103: 937-943, 2008.

8. Dijkmans B and Gerards A: Cyclosporin in rheumatoid arthritis: Monitoring for adverse effects and clinically significant drug interactions. BioDrugs 10: 437-445, 1998.

9. Stang A: Critical evaluation of the Newcastle-Ottawa scale for the assessment of the quality of nonrandomized studies in meta-analysis. Eur J Epidemiol 25: 603-605, 2010.

10. Jadad AR, Moore RA, Carroll D, Jenkinson C, Reynolds DJ, Gavaghan DJ and McQuay HJ: Assessing the quality of reports of randomized clinical trials: Is blinding necessary? Control Clin Trials 17: 1-12, 1996.

11. Ehrén I, Hallén Grufman K, Vrba M, Sundelin R and Lafolie P: Nitric oxide as a marker for evaluation of treatment effect of cyclosporine A in patients with bladder pain syndrome/interstitial cystitis type 3C. Scand J Urol 47: 503-508, 2013.

12. Chade J, Chade D, Lucon AM, Bruschini H and Srougi M: 462 5-year follow-up of patients with refractory interstitial cystitis treated with cyclosporine A: A prospective single-institution study. Eur Urol (Supp 13): e462, 2014.

13. Forrest JB, Payne CK and Erickson DR: Cyclosporine A for refractory interstitial cystitis/bladder pain syndrome: Experience of 3 tertiary centers. J Urol 188: 1186-1191, 2012.

14. Sairanen J, Forsell T and Ruutu M: Long-term outcome of patients with interstitial cystitis treated with low dose cyclosporine A. J Urol 171: 2138-2141, 2004.

15. Forsell T, Ruutu M, Isoniemi H, Ahonen J and Alfthan O: Cyclosporine in severe interstitial cystitis. J Urol 155: 1591-1593, 1996.

16. Sairanen J, Tammela TL, Leppilahti M, Multanen M, Paananen I, Lehtoranta K and Ruutu M: Cyclosporine A and pentosan polysulfate sodium for the treatment of interstitial cystitis: A randomized comparative study. J Urol 174: 2235-2238, 2005.

17. Sairanen J, Hotakainen K, Tammela TL, Stenman UH and Ruutu M: Urinary epidermal growth factor and interleukin-6 levels in patients with painful bladder syndrome/interstitial cystitis treated with cyclosporine or pentosan polysulfate sodium. Urology 71: 630-633, 2008.

18. Sairanen J, Tammela TL, Leppilahti M, Onali M, Forsell T and Ruutu M: Potassium sensitivity test (PST) as a measurement of treatment efficacy of painful bladder syndrome/interstitial cystitis: A prospective study with cyclosporine A and pentosan polysulfate sodium. Neurourol Urodyn 26: 267-270, 2007.

19. Gao X, Ma Y, Sun L, Chen D, Mei C and Xu C: Cyclosporine A for the treatment of refractory nephritic syndrome with renal dysfunction. Exp Ther Med 7: 447-450, 2014. 